\title{
Lineage and fate of each blastomere of the eight-cell sea urchin embryo
}

\author{
R. Andrew Cameron, Barbara R. Hough-Evans, Roy J. Britten, ${ }^{1}$ and Eric H. Davidson \\ Division of Biology, California Institute of Technology, Pasadena, California 91125 USA
}

\begin{abstract}
A fluoresceinated lineage tracer was injected into individual blastomeres of eight-cell sea urchin (Strongylocentrotus purpuratus) embryos, and the location of the progeny of each blastomere was determined in the fully developed pluteus. Each blastomere gives rise to a unique portion of the advanced embryo. We confirm many of the classical assignments of cell fate along the animal-vegetal axis of the cleavage-stage embryo, and demonstrate that one blastomere of the animal quartet at the eight-cell stage lies nearest the future oral pole and the opposite one nearest the future aboral pole of the embryo. Clones of cells deriving from ectodermal founder cells always remain contiguous, while clones of cells descendant from the vegetal plate (i.e., gut, secondary mesenchyme) do not. The locations of ectodermal clones contributed by specific blastomeres require that the larval plane of bilateral symmetry lie approximately equidistant (i.e., at a $45^{\circ}$ angle) from each of the first two cleavage planes. These results underscore the conclusion that many of the early spatial patterns of differential gene expression observed at the molecular level are specified in a clonal manner early in embryonic sea urchin development, and are each confined to cell lineages established during cleavage.
\end{abstract}

[Key Words: Development; cell lineage; dye injection; axis specification]

Received December 8, 1986; accepted December 24, 1986.

Though it may appear outwardly a simple hollow sphere of undifferentiated, ciliated cells, the early blastula-stage sea urchin embryo is already a spatially organized mosaic of differentially functioning, multicellular regions. Each region is composed of a particular set of clones descendant from early cleavage founder blastomeres. In some regions the ultimate cell fate is definitively specified by the blastula stage, and the cells have begun to express particular sets of genes (for review, see Davidson 1986). Thus, for example, in the 100- to 200-cell blastula of Strongylocentrotus purpuratus the CyIIIa cytoskeletal actin gene and the Spec1 $\mathrm{Ca}^{2+}$-binding protein gene have already been activated in the precursors of the aboral ectoderm cells, to which their transcripts are confined in advanced embryos (Lynn et al. 1983; Angerer and Davidson 1984; Cox et al. 1986). At the blastula stage these precursors are located mainly in one hemisphere of the embryo. Similarly, a gene coding for a protein later found on the surfaces of skeletogenic mesenchyme cells that may be involved in $\mathrm{Ca}^{2+}$ transport and a skeletal matrix protein gene, both of which are active during embryogenesis exclusively in the skeletogenic mesenchyme cells, have begun to be transcribed in cells of this lineage even while they are still embedded in the

${ }^{1}$ Also Staff Member, Carnegie Institute of Washington. vegetal blastocoel wall (Carson et al. 1985; Benson et al. 1987; Sucov et al. 1987). Following the ingression of the skeletogenic mesenchyme cells there remains at the base of the blastula a disc of pluripotential blast cells, known as the vegetal plate, from which there later derive a number of other differentiated cell types. These include cells required for construction of stomach and intestine, muscle, pigment cells, and portions of the coelomic sacs. Molecular markers of differentiation in vegetal plate derivatives usually appear later in development, during or after gastrulation. Among these are transcripts of the genes coding, respectively, for muscle actin and muscle myosin heavy chain (Cox et al. 1986; S.J. Rose et al., in prep.); and proteins recognized by monoclonal antibodies that occur specifically in various regions of the gut and in pigment cells (McClay et al. 1983; Gibson and Burke 1985; Cox et al. 1986; S.J. Rose et al., in prep.). It has been known since the initial observations and fate map experiments of Boveri (1901) and Hörstadius (review, 1939) that the vegetal plate derivatives, the skeletogenic mesenchyme, and portions of the ectoderm each descend from specific tiers of blastomeres along the animal-vegetal axis of the early cleavage embryo. Thus, in a general sense the patterns of differential gene expression in this embryo are often, if not always, instituted in a lineage-specific fashion, in that these patterns are often segregated to different blastomere lineages and at least in some cases do not appar- 


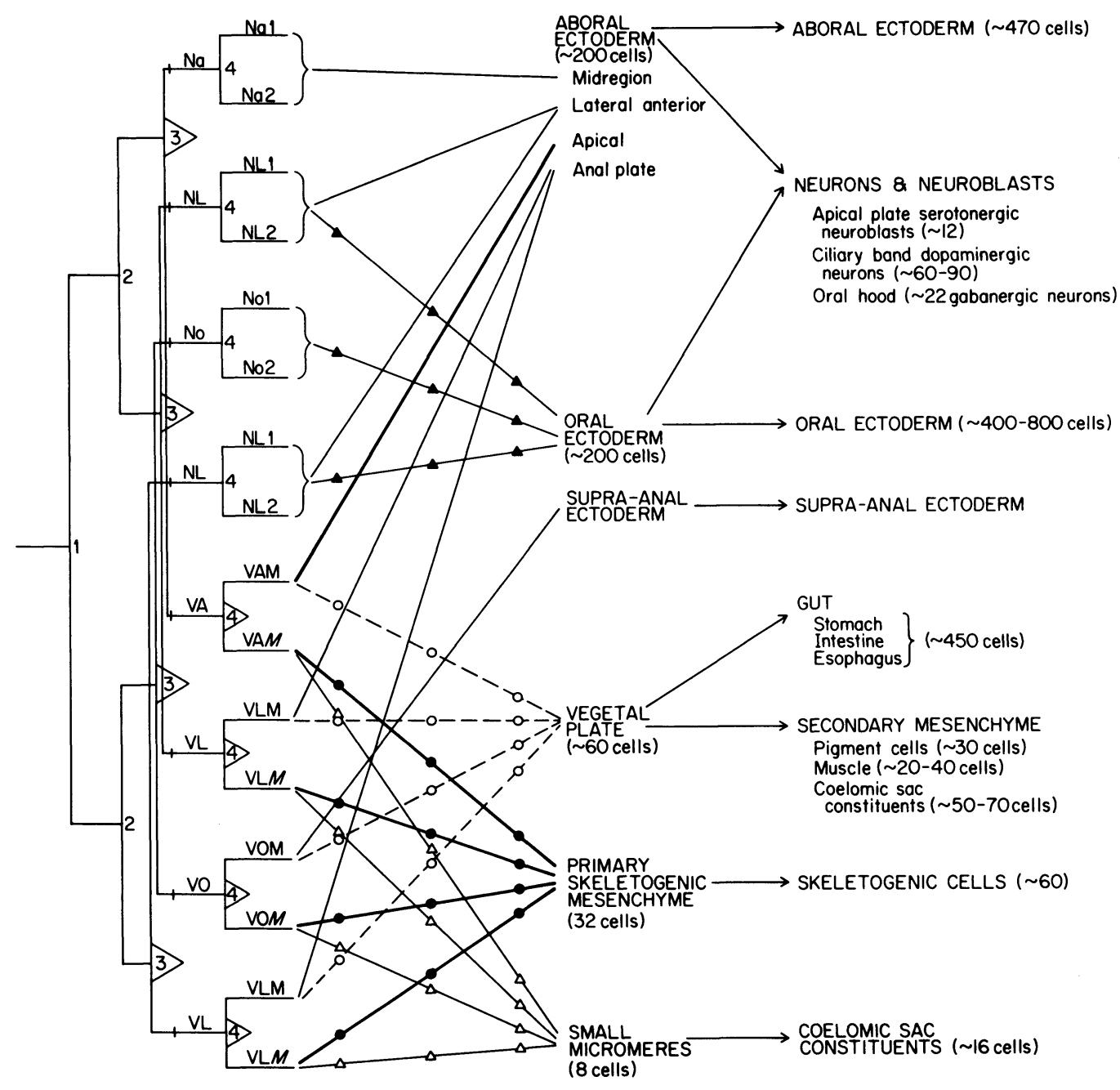

Figure 1. A diagram of the first four cleavages in the sea urchin embryo illustrating cell fates at the mesenchyme, blastula, and pluteus stages. Although the timing differs among species, the patterns of the early lineage, the sequence of division cycles, and the cell fates are the same among those species that have been studied (for review, see Okazaki 1975). This is a simplified version of the diagram published in Davidson (1986), wherein can be found a complete discussion of its derivation. The symbols used are as follows: $(A, a)$ aboral; (L) lateral; $(M)$ macromere; $(M)$ micromere; $(N)$ animal; $(\mathrm{O}, 0)$ oral; $(V)$ vegetal. In the nomenclature used, wholly capital designations indicate founder cells that will give rise to cell lineages of several diverse fates, as perceived at the late (mesenchyme) blastula stage, while cell names including a lower-case letter designate a progenitor of a clone of cells of identical fate (so far as is known). Thus, for example, all the descendants of the $\mathrm{Na}$ blastomere give rise exclusively to aboral ectoderm cells (see Fig. 4.5 of Davidson 1986). Each horizontal line represents a cell and each vertical line a division. The number of each cleavage (i.e., first-fourth) is given, and those divisions in which the cleavage plane is horizontal are marked with a triangle around the cleavage number. The first two cleavage planes are vertical (i.e., parallel to the animal-vegetal axis, or meridional). The third cleavage plane is horizontal (perpendicular to the animal-vegetal axis, or equatorial). In the animal quartet, the fourth cleavage is vertical producing the mesomeres ( $\mathrm{Na} 1, \mathrm{Na} 2$, the two NL1 and two NL2 cells, and No1 and No2). In the vegetal quartet, the fourth cleavage is horizontal and unequal, producing the macromeres (VAM, the two VLM cells, VOM) and the micromeres (VAM, the two VLM cells, and VOM). In the next two cleavages the macromeres first divide vertically and then horizontally, so that at the sixth division clones of the lower tier of cells contribute exclusively to the vegetal plate [named the $v e g_{2}$ lineage by Hörstadius $(1939,1973)$ ] and clones of the upper tier of cells contribute exclusively to the ectoderm [called the veg lineage by Hörstadius $(1939,1973)$ ]. Approximate cell numbers for each cell type later in development are indicated in parentheses. 
ently cross their boundaries (Angerer and Davidson 1984; for review, see Davidson 1986).

Recently a detailed lineage map for the early sea urchin embryo through six to seven cleavages has been constructed from literature sources (A. Cameron and E. Davidson; see Davidson 1986 for map, nomenclature, sources, and discussion). The map indicates specific origins for the later cell types of the embryo, though because it relies in part on data from several species it is for some regions of the embryo a canonical rather than exact map of the later cleavages/cleavage terminates after 9-10 divisions). A simplified version extending only through fourth cleavage is shown in Figure 1. This period is well known and the regions of the map shown can be regarded as exact. The central column of Figure 1 displays the cell types of the mesenchyme blastula, and the right-hand column the respective differentiated derivatives of the completed pluteus-stage embryo. Figure 1 follows classical sources in respect to many of the assignments along the animal-vegetal axis, as detailed in the legend. The animal-vegetal axis is of maternal origin in the sea urchin egg and can be observed prior to fertilization (Boveri 1901; Schroeder 1980). Results from ablation experiments, culture of partial embryos, and other kinds of evidence (for review, see Davidson 1986) indicate a preformed, differential localization of morphogenetic potentialities along this axis. However, the origin of the oral-aboral axis of the embryo so far remains obscure. Morphologically, the future oral side of the embryo is not revealed until early in gastrulation when the skeletogenic mesenchyme cells aggregate in bilateral clusters, at the oral side of the base of the invaginating archenteron. Ultimately, the archenteron bends toward the ectodermal wall on this side, and the mouth is formed at the site of contact (Gustafson and Wolpert 1963). Since, as noted above, even in early blastula-stage embryos the progenitors of the aboral ectoderm express a set of genes not transcribed in the progenitors of the oral ectoderm, initial specification of the oral-aboral axis must occur during if not before cleavage. There is no convincing, or direct, evidence that the oral-aboral axis is preformed in the egg, as is the animal-vegetal axis (though this has been argued by Hörstadius and Wolsky 1936), and reversal of the animal pole blastomere cap shows that this axis can be respecified far into cleavage (for review, see Hörstadius 1973). However, Czihak (1963) demonstrated that those animal and vegetal blastomeres of eight-cell-stage Paracentrotus embryos destined to give rise to ectoderm on the future oral side stain differentially for cytochrome oxidase. This key observation implies that in undisturbed embryos the oral-aboral axis has in fact been specified by the eightcell stage. Implicit in the fate assignments shown in the lineage map of Cameron and Davidson (Davidson 1986), and in the simplified version shown in Figure 1, are the assumptions that the future oral-aboral axis is in fact defined by this stage, and that specific blastomeres located oppositely in the embryo will give rise to the polar ectodermal regions of this axis. The experiments described herein demonstrate these propositions directly.
In addition, we determine for the first time the lineal origins of all regions of the embryonic ectoderm, correcting various misapprehensions at least a half century old; we determine the relation between the plane of bilateral symmetry and the first two cleavage planes; and we confirm in general the assignments of blastomere cell fate along the animal-vegetal axis that are shown in Figure 1.

\section{Results}

\section{Technical aspects}

About $50 \%$ of embryos labeled by injection of fluoresceinated dextran into a blastomere at the eight-cell stage developed normally. Pluteus-stage embryos that contained fluorescein-labeled cells but displayed any detectable morphological imperfections were excluded from the analysis. A frequently occurring abnormality was the presence of a single large cell, either fluorescent or dark, remaining in the blastocoel of an otherwise normal pluteus embryo. Sometimes such cells seemed to undergo cycles of division on a schedule much retarded from the normal one. Clumps of cells lying in inappropriate locations in the blastocoel were sometimes also observed. In the method of injection that we utilized, the solution flows continuously from the orifice of the microneedle. Thus, the amount injected depended on the dwell time of the needle within the blastomere, which could not be perfectly controlled. Methodological experiments indicated that the $\cong 50 \%$ toxicity observed probably resulted from delivery of too large a quantity of the fluoresceinated lineage tracer.

\section{Patterns of labeling observed}

Six discrete patterns of fluorescein-stained cells were found in the normally developing labeled plutei. Ectodermal labeling was always limited to one congruent patch of cells, but in the gut several separate patches were sometimes apparent, and individual mesenchyme cells were labeled. In the aboral ectoderm, scattered dark cells in the midst of a patch derived from an injected animal blastomere were invariably pigment cells. The pigment does not quench the fluorescence per se, since these cells were found to be labeled when included in labeled ectodermal patches derived from vegetal blastomeres. Pigment cells have been shown to be a product of the secondary mesenchyme (Gibson and Burke 1985), and are thus among the derivatives of the vegetal plate formed from descendants of vegetal blastomeres (cf. Fig. 1).

In three of the six patterns observed, ectoderm and vegetal plate derivatives (i.e., gut and mesenchyme) were both labeled. From the vital staining experiment of Hörstadius, von Ubisch, and others (for review, see Hörstadius 1973), these patterns indicate that the fluoresceinated lineage tracer had been injected into members of the vegetal tier of blastomeres. Labeling patterns that were confined to ectoderm indicated that the dye had been injected into one of the four animal pole blasto- 


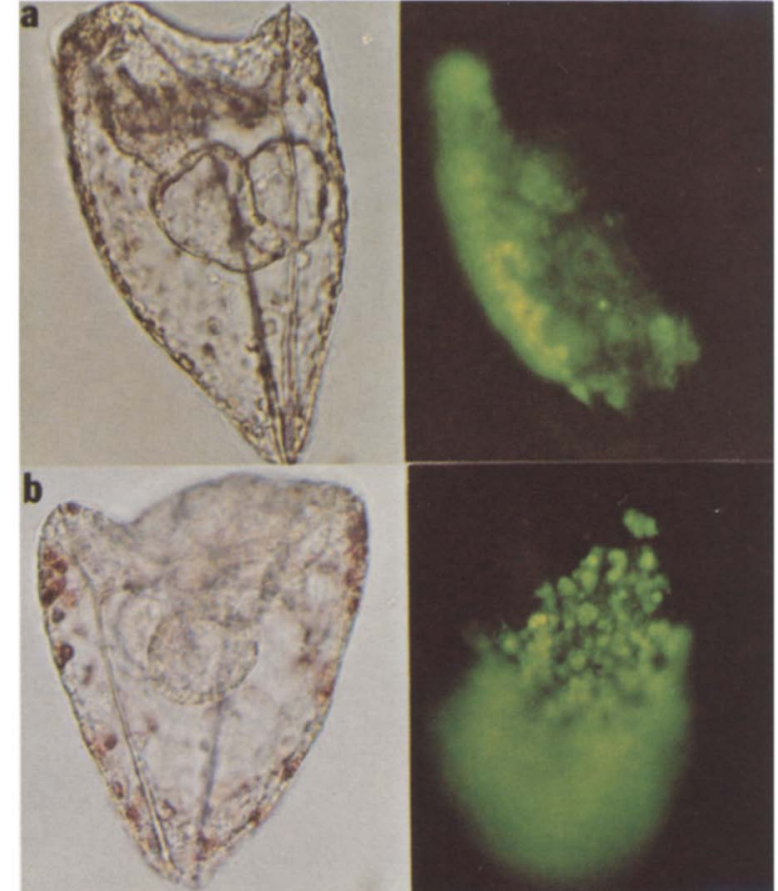

Figure 2. The $\mathrm{Na}$ pattern. (a) Whole mount of a pluteus viewed from the side with the anus to the right and the mouth at the top. The esophagus, stomach, and expanded intestine are clearly visible through the transparent ectoderm. The right body rod and right anterolateral rod of the larval skeleton lie in the plane of focus. With epifluorescence, a region of the aboral ectoderm extending from the ciliated band above the mouth in the aboral direction about two-thirds of the way to the apex is illuminated. There is no labeling in the gut. $(b)$ Whole mounts of a pluteus viewed from the side opposite the anus with the plane of focus on the midregion of the aboral ectoderm. The body rod and the anterolateral rod lie in focus to the left of the gut. The fluorescently labeled cells comprise the midregion extending from the ciliated band to about two-thirds of the way to the aboral apex.

meres. The embryo develops in a bilaterally symmetrical manner, and equivalent patterns are formed by either of the two lateral blastomeres of the eight-cell embryo, i.e., the two VL blastomeres in the vegetal tiers, and the two NL blastomeres in the animal tier. The remaining blastomeres on the future oral side, designated in Figure $1 \mathrm{VO}$ and No, and on the future aboral side, VA and $\mathrm{Na}$, each produce a unique labeling pattern. These six patterns are now illustrated, and described in further detail.

Na pattern The Na pattern of fluorescent label covers the midregion of the aboral ectoderm, as shown in Figure 2. Anteriorly, this region reaches to the ciliated band above the mouth. It extends in the aboral direction almost, but not completely, to the apex. Laterally, the labeled cells extend down the sides and include more

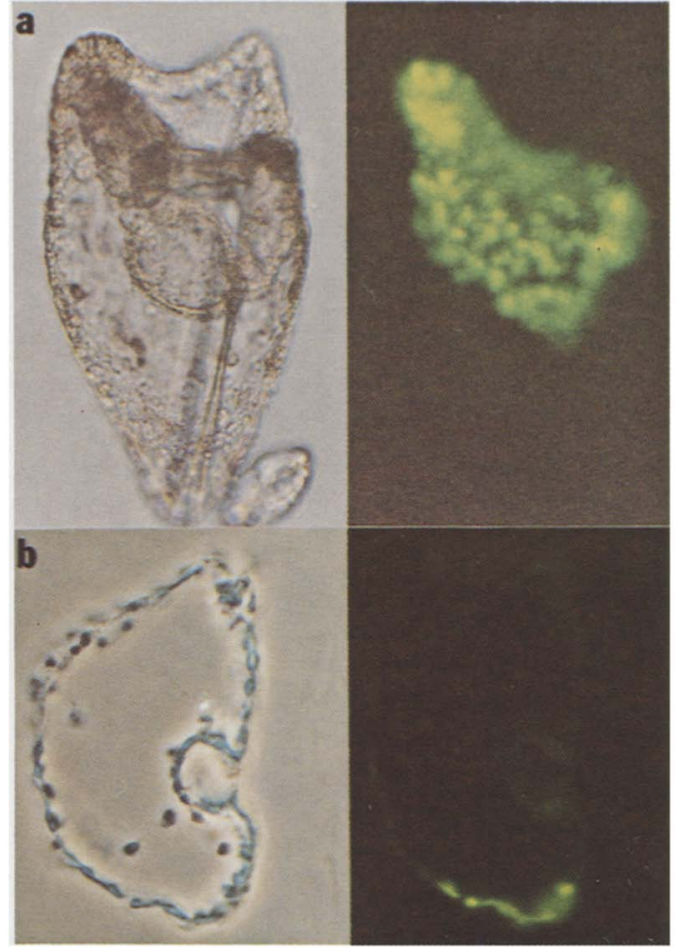

Figure 3. The NL pattern. (a) Whole mount of a pluteus viewed from the side with the anus to the right and the mouth at the top. The nearest postoral and anterolateral arms /right side of embryol are in focus. The small body attached to the aboral apex is debris. In epifluorescent illumination, a region of the aboral ectoderm extending onto both arms is labeled. $(b)$ Section of a pluteus that passes obliquely through the oral and aboral ectoderm at the level of the mouth. The flatter oral ectoderm is to the right. Although both lateral aboral ectodermal regions are in the section, fluorescent cells are only found in one lateral aboral ectoderm, the contiguous ciliated band, and oral ectoderm.

than one-third of the aboral ectoderm. No dyed cells are found in the gut, among secondary mesenchyme cells, or the primary mesenchyme cells that lie along the spicules.

$N L$ pattern Each NL pattern exhibits fluorescent label in a region of the ectoderm that reaches across the ciliated band on one side of the pluteus, as illustrated in Figure 3. This region, called the lateral ectoderm, extends anteriorly to the tip of the rudimentary anterolateral arm and in the aboral direction about one-fourth of the distance to the apex. On the anal side, the labeling extends onto the postoral arm. Neither the gut nor any of the mesenchyme cells are labeled.

No pattern The No pattern includes a large portion of the oral ectoderm and no other tissue in the pluteus, as 


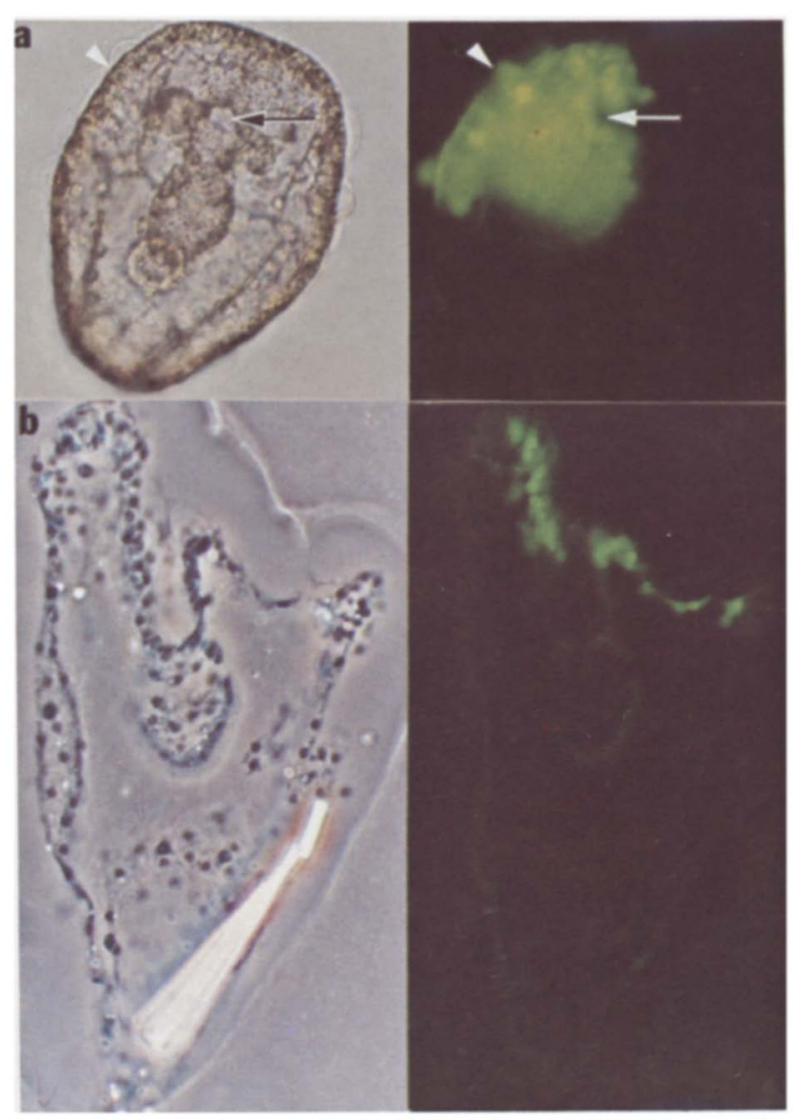

Figure 4. The No pattern. (a) Whole mount of a pluteus in facial view with the plane of focus at the oral ectoderm. The mouth (arrow) lies in the plane of focus and obscures the esophagus, though the coelomic pouches at either side of the esophagus can be discerned. The tips of the postoral skeletal rods are in focus above the mouth. The stomach and intestine extend downward toward the aboral apex. The anus can be seen between the body rods. Epifluorescent illumination reveals a group of labeled cells extending above and below the mouth (arrow) and into the lateral ciliated band. An arrowhead indicates a point of correspondence between the bright field and epifluorescent images. (b) Sagittal section of a pluteus. The plane of section passes through the oral ectoderm and the esophageal lumen, and one wall of the stomach. One highly refractile body rod lies in the section. Only the cells of the oral ectoderm display the fluorescent label.

shown in Figure 4. The remainder of the oral ectoderm is contributed by progeny of the NL cells. In section, the labeled cells can be seen to lie both above and below the mouth but do not extend into the esophagus. Neither the aboral ectoderm, the gut, nor the mesenchyme cells are labeled.

VL pattern The gut, mesenchyme cells, and, for each VL cell, one side of the anal plate aboral ectoderm are labeled in this pattern, as illustrated in Figure 5. The median oral-aboral boundary of the labeled anal plate ectoderm formed by progeny of each VL cell intersects the

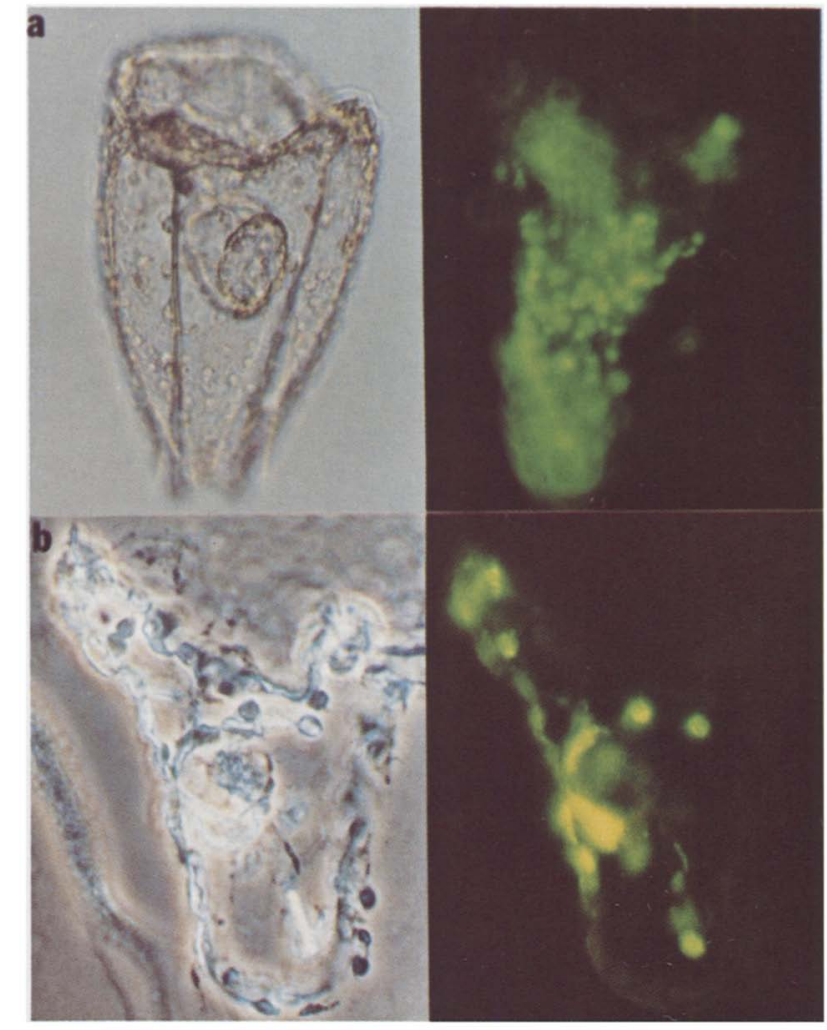

Figure 5. The VL pattern. (a) Whole mount of a pluteus seen from the anal side with the anal plate of the aboral ectoderm in focus. The intestine and the right body rod with attached skeletogenic mesenchyme cells are also in the plane of focus. Fluorescently labeled cells are found amongst the mesenchyme cells, in the gut, and on one side of the anal plate aboral ectoderm. Because the ectoderm is curved and the overlying ectoderm refracts the epifluorescence, the extreme edges of the fluorescent regions are blurred. (b) Sagittal section of a pluteus, including one anterolateral arm (to the right) and one postoral arm. Some but not all of the mesenchyme cells, some of the gut cells, and the anal plate aboral ectoderm cells contain fluorescent label.

anus. The labeled ectoderm cells do not extend to the aboral apex of the embryo nor anteriorly to the ciliated band. Some, but not all, of the skeletogenic mesenchyme cells that can be identified along the skeletal rods contain the fluoresceinated tracer.

VA pattern Portions of the gut, some mesenchyme cells, and all of the most posterior portion of the aboral ectoderm display fluorescence, as shown in Figure 6. The labeled ectoderm forms a hollow cone with the extended body rods at the apex. Some single secondary mesenchyme cells and skeletogenic mesenchyme cells in the blastocoel associated with the spicules are labeled. The labeling in the gut may form from one to several patches. Labeling at the tips of the larval arms is restricted to the mesenchyme cells and is not found in the ectoderm. 
VO pattern The gut, mesenchyme cells, and the ectoderm between the mouth and the anus are labeled, as shown in Figure 7. The aboral boundary of the labeled ectoderm extends to and includes the upper edge of the anus. It extends laterally near the anterolateral arms and anteriorly into the oral ectoderm. Observations on sections (not shown) establish conclusively that the supraanal ectoderm contains the fluoresceinated label. No pattern is apparent in the arrangement of those mesenchyme cells that are labeled.

\section{Distribution of labeling patterns in the sample}

The blastomeres of the living eight-cell $S$. purpuratus embryo cannot be distinguished visually, at least at light microscope resolution, and thus we did not know in advance which was being injected with the fluoresceinated dextrans. Their identification ex post facto followed from analysis of the staining patterns observed in each case at the pluteus stage, as illustrated in Figures $2-7$. Had the injections been equally successful for each blastomere type, and were there no systematic bias in choice of blastomere for injection, the distribution of the patterns expected would be $(1 \mathrm{Na}: 2 \mathrm{NL}: 1 \mathrm{No})$ : (1Va : $2 \mathrm{VL}:$ 1VO). A total of 102 labeled pluteus-stage embryos were scored in this study, and Table 1 shows the ratios obtained. Although these do not coincide exactly with the expected ratios, they are not significantly different from random expectations, according to a $\chi^{2}$ analysis (Snedecor and Cochran 1979). This suggests that on the assumption of the ratios expected the probability of obtaining the ratios as different from expectation as observed in our sample falls in the range of $10-25 \%$.

\section{Discussion}

\section{Cell lineage of the embryonic ectoderm}

The regions of the ectoderm in the completed pluteusstage embryo that derive from each of the blastomeres of the eight-cell embryo are shown diagrammatically in Figure 8. The two general characteristics of the fluorescent labeling patterns that underlie this interpretation

Table 1. Distribution of labeling patterns in all pluteus-stage embryos included in this study

\begin{tabular}{lc}
\hline Labeling pattern & Number observed \\
\hline $\mathrm{Na}$ & 13 \\
$\mathrm{NL}$ & 19 \\
$\mathrm{No}$ & 7 \\
$\mathrm{VA}$ & 14 \\
$\mathrm{VL}$ & 29 \\
$\mathrm{VO}$ & 20 \\
$\quad$ Total & 102 \\
\hline
\end{tabular}

are that each of the six patterns observed is unique, and that, as noted above in each pattern, the labeled cells of the ectodermal wall always form a single coherent and contiguous patch. Widespread ectodermal cell migration, or spatial mixing of the progeny of different clones, evidently does not occur in the course of embryonic development. Labeled mesenchyme cells (and their derivatives) are, in contrast, not contiguous to one another, as expected from their independent migratory behavior. The careful observations of Ettensohn (1985) and Hardin and Chen (1986) have shown that rearrangement of cells with respect to one another also occurs during the invagination of the archenteron, and this probably accounts for the multiple, separated distribution of labeled cell patches in the gut of the pluteus (e.g., Fig. 5b). Though the six ectodermal labeling patterns we observed are at least to a first approximation mutually complementary and nonoverlapping, we cannot be certain to what extent the clonal boundaries might vary from embryo to embryo, i.e., with respect to the overall morphological coordinates of the pluteus. In Figure 8 we illustrate the approximate reproducibility of these ectodermal staining patterns, here shown in three indpendent examples of plutei that are labeled in an NL cell. The simplest of these boundaries to orient is that separating the lateral halves of the anal plate ectoderm, constructed respectively by the progeny of the two VL cells. Our impression is that this boundary adheres generally to the vertical midline of the embryo on that ectodermal face, i.e., to a line drawn anteriorly from the aboral apex of the embryo through the middle of the anus. However, the edges of these clones appear on a fine scale to interdigitate, in that they do not usually form a perfectly smooth seam. Some clonal admixture may also occur in the ciliated band. As shown in the foregoing fluorescence photomicrographs, progeny of the NL blastomeres as well as of No contribute to this structure, and on the anal side, progeny of VO appear to be involved as well. We are not certain to what extent the progeny of No, which form the main area of the facial ectoderm, are at its edges intermingled with descendants of the NL and VO blastomeres in the thickened ciliated band structure. The origin of the ciliated band is of particular interest since it is here that a particular set of neuroblasts arises late in postgastrular development (R. Burke, pers. comm.).

The clonal ectoderm assignments summarized in Figure 9 differ in essential respects from those proposed classically. Thus, the tier of eight sixth-cleavage ectodermal founder cells arising from the four vegetal macromeres (i.e., veg $g_{1}$ of Hörstadius 1939) do not all give rise to anal plate ectoderm. Hörstadius (review, 1973) assumed this on the basis of experiments in which individual blastomeres of this tier were stained and the location of their progeny determined in the embryo. Instead, as illustrated in Figure 9, descendants of VA contribute the apical aboral ectoderm on all sides of the embryo; the anal plate is the product only of the VL ectodermal clones; and the supra-anal ectoderm, and the edge of the oral ectoderm on that side, derived from the VO ecto- 
dermal clones. Nor is the distribution in the ectoderm of the animal tier blastomere progeny summarized in Figure 9 indicated in previous descriptions.

Figure 10 illustrates our concept of the progressive disposition of these ectodermal clones during development. This diagram provides a stepwise description of the effects of elongation along the oral-aboral axis, as the skeletal body rods extend in the aboral direction. In the completed pluteus these rods remain anchored at the vertex within the domain of the VA progeny, as can be seen in Figure 6.

\section{The initial oral-aboral axis}

As shown in Figure 9 the oral-aboral axis of the pluteus-stage embryo lies in a plane of bilateral symmetry that bisects the mouth, the anus, the anal plate ectoderm, and on the opposite side the midregion ectoderm descendant from the $\mathrm{Na}$ blastomere. At the oral end of this plane is the face, formed of the clone of cells generated by the No progenitor cell, and at the aboral end is located the apical clone of ectoderm cells descendant from VA. In the nearly spherical gastrula the No and VO ectodermal clones and the $\mathrm{Na}$ and VA ectodermal clones are positioned opposite one another (Fig. 9). Therefore, in the eight-cell embryo the initial plane of bilateral symmetry, and the oral-aboral axis, must bisect the No and VO blastomeres on the future oral side, and the $\mathrm{Na}$ and VA blastomeres on the future aboral side. Viewed from the animal pole of the egg, it follows that the future plane of bilateral symmetry and the oral-aboral axis probably lie at a $45^{\circ}$ angle from the first and second cleavage furrows. These cleavages, which separate the oral and aboral blastomeres from the adjacent lateral blastomeres, pass vertically along the animal-vegetal axis of the egg, and are always orthogonal to one another. It remains to be determined when this axis is initially specified. Our present results show only that by the time third cleavage has been completed there have been formed blastomeres, the progeny of which give rise exclusively to oral and aboral ectoderm, respectively. However, the initial axial specification could have occurred at second cleavage, or even prior to cleavage, though the polarized staining observed by Czihak (1963) at the eight-cell stage was not detected earlier. Furthermore, Hörstadius and Wolsky (1936) found no consistent correlation between the position of the first cleavage plane and the orientation of the axes of the later embryo.

Cell lineage and early pattern of gene expression in the sea urchin embryo

The two early differentiating cell types of the sea urchin embryo for which the greatest amount of molecular evidence exists (see introductory section) are the skeleto- genic mesenchyme and the aboral ectoderm. The skeletogenic mesenchyme provides a clear example of a more or less autonomously differentiating, clonal, embryonic lineage. At the fifth cleavage four progenitor cells segregate from the micromeres (i.e., VAM, the two VLM cells, and VOM; Fig. 1); these divide three times further, and after ingression into the blastocoel the 32 progeny then embark on the morphogenetic and biochemical activities required for skeletogenesis. Skeleton formation occurs in vitro, on an approximately normal schedule, when micromeres are isolated and cultured under appropriate conditions (Okazaki 1975; Harkey and Whiteley 1980; Carson et al. 1985). We show here that the aboral ectoderm also displays a discrete, clonal lineage, though the details of the later cleavages are known only in canonical fashion (Davidson 1986). However, the fates of the ectoderm progenitor cells can be altered in ectopic blastomere transplantation experiments, and the polarity of the oral-aboral axis remains plastic into late cleavage (for review, see Hörstadius 1973). Perhaps this indicates that early blastomere interactions are involved in the polarization process by which cell fate is specified along the oral-aboral axis, while the self-differentiation of the skeletogenic mesenchyme lineage depends to a larger extent on inheritance of maternal factors normally distributed to the vegetal region of the egg and sequestered into the micromeres at fourth cleavage. In any case, it is clear that in the undisturbed embryo the $\sim 200$ aboral ectoderm cells of the blastula and the $\sim 470$ of these cells in the advanced pluteus derive clonally from six specific progenitor cells (see Fig. 1). That cells of the different clones constituting the aboral ectoderm all express a particular set of genes, including the CyIIIa and Spec1 genes, is clear from comparisons of the in situ hybridization patterns using these probes (Lynn et al. 1983; Angerer and Davidson 1984; Cox et al. 1986) with the lineage diagram shown in Figure 1 of this paper. One of the six clonal progenitor cells is $\mathrm{Na}$, since all of its progeny become aboral ectoderm. The descendants of the two NL cells that give rise exclusively to aboral ectoderm probably arise at the vertical fourth cleavage, as this is the simplest interpretation of the spatial positions occupied by their descendants (Figs. 9 and 10). The three clonal progenitors of the aboral ectoderm descendant from VA, and from the two VL cells, almost certainly arise at sixth cleavage, when they are segregated from vegetal plate progenitors.

The significance of the polyclonal origin of the aboral ectoderm can be viewed in different ways that are subject to experimental test. At one extreme, it is conceivable that a genomic "switch" is thrown in the six clonal progenitors of the aboral ectoderm that potentiates activation of genes required for aboral ectoderm differentiation, and that the state of this switch is propagated to the clonal derivatives of these progenitors. At the other, the significance of the polyclonal aboral ectoderm lineage might be only that it is a spatial device whereby the appropriate cytoplasm is ultimately divided up amongst the aboral ectoderm cells. This cytoplasm could contain trans-activators required for aboral ectoderm gene ex- 


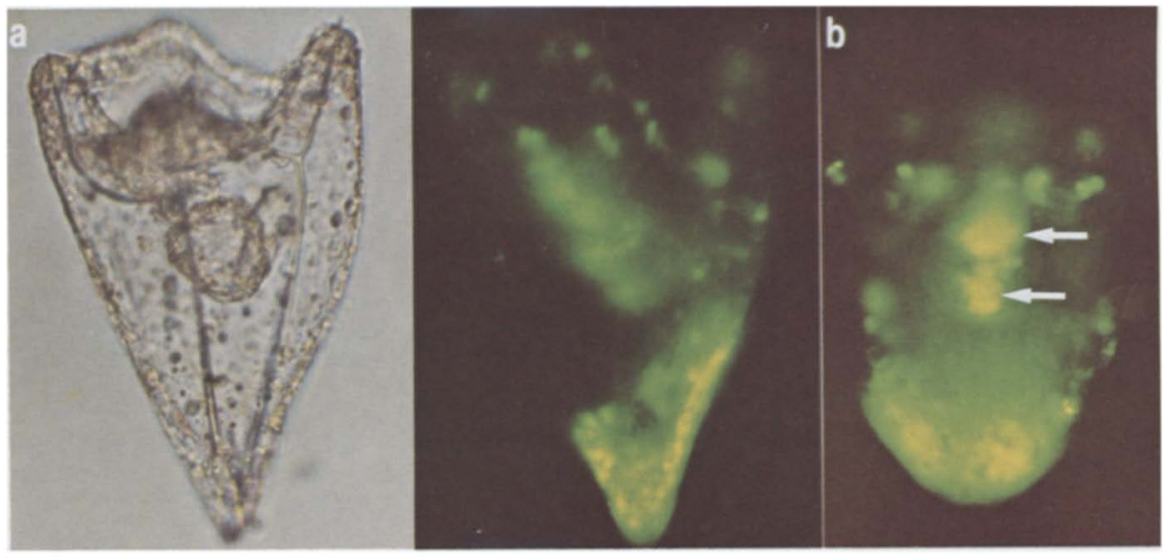

Figure 6. The VA pattern. (a) Whole mount of a pluteus viewed obliquely from the side, mouth to the left. The plane of focus lies midway through the body so that one anterolateral and the opposite postoral arm are in focus. The anterior end of the left body rod and the junction with the anterolateral rod are also in focus below the gut. Fluorescently labeled cells are found among the mesenchyme cells, in the gut, and in the apical aboral ectoderm. $(b)$ Epifluorescent image of a different pluteus viewed from the anal side. This illustrates the noncontiguous patches (arrows) of labeled cells found in the gut.

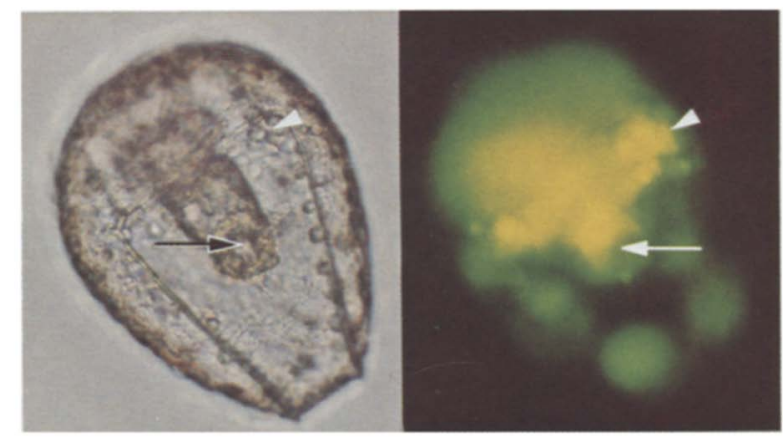

Figure 7. The VO pattern. Whole mount of pluteus viewed from the anal side. The supra-anal ectoderm, the ciliated band below the mouth, and the body rods near the junction with the anterolateral rods are all in focus. Fluorescently labeled cells are found amongst the mesenchyme cells, in the gut, and in the ectoderm anterior to the anus, where they extend into the oral ectoderm. The most brightly fluorescent cells that are in the plane of focus are ectoderm cells extending forward from the anus (arrow) and laterally between the anterolateral arms. The images of the skeletogenic mesenchyme cells are blurred by refraction. An arrowhead indicates a point of correspondence between the bright-field and epifluorescent images.

pression. The spatial contiguity of the aboral ectoderm clones observed in this study, the lack of early cell migration in the aboral ectoderm, and the close retention of the initial relative positions of these clones at the time differential aboral ectoderm gene expression is activated in the early blastula are consistent with either alternative. However, it is a striking though obvious point that each of the six aboral ectoderm clones must in the aggregate contain more or less the same cytoplasm as did its single early-cleavage progenitor.

We cannot yet appreciate the extent to which embryonic lineage considerations will provide a guide to patterns of gene function in the sea urchin embryo. They are unlikely to be directly relevant to the process by which the vegetal plate blast cells assume their diversi- fied functional characteristics at and after gastrulation. There are a number of genes known, furthermore, that are activated during development in many unrelated cell lineages, e.g., the CyI actin gene (Cox et al. 1986), one of the metallothionein genes (Angerer et al. 1986), and the calmodulin gene (Floyd et al. 1986). Certain examples suggest, however, that lineage may prove relevant to patterns of gene expression occurring later than might a priori have been supposed. Thus, for example, McClay et al. (1983) described a monoclonal antibody that reacts with a protein found in the hind gut and the flanking anal ectoderm, a pattern that would be explained if this protein were produced only in descendants of the VL cells. Another possible example concerns a homeo box gene, transcripts of which have been localized in 
pluteus-stage embryos by Dolecki et al. (1986). In the late pluteus these transcripts are confined to a patch of apical aboral ectoderm cells that appear to be distributed
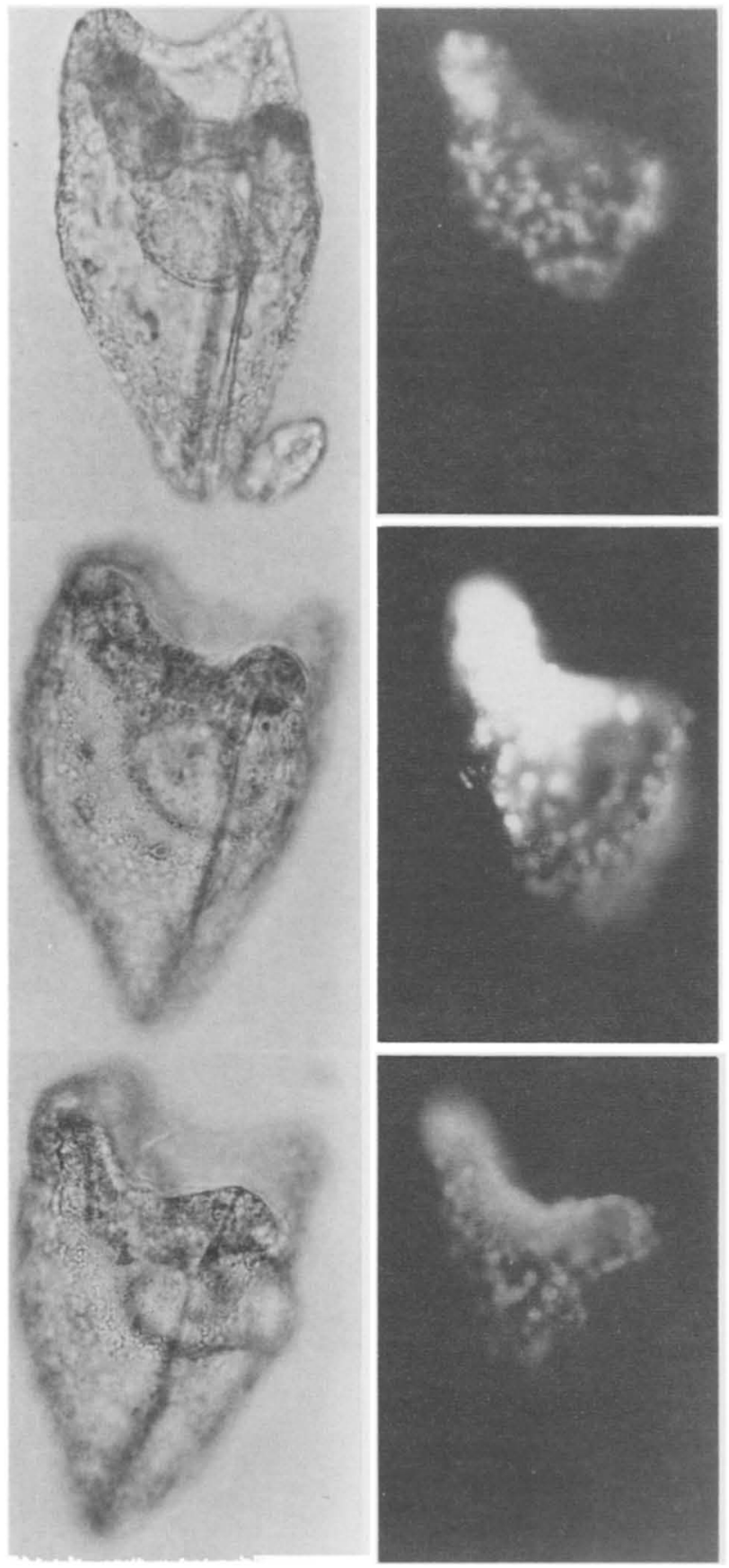

Figure 8. Three different examples of the NL pattern to illustrate the approximate reproducibility in the location of this clone of labeled ectodermal cells. The plutei are viewed from the right side with the face upward and the anus to the right. The uppermost example is the same as Fig. 3. The photograph of the second embryo is overexposed to display the margins of the pattern. The plane of focus in both the bright-field and fluorescent images lies on the ciliated band. to almost exactly the same region of the embryo as is the clone of ectodermal cells descendant from the VA blastomere (Figs. 6 and 9).

In the early sea urchin embryo the cell lineages provide a means for generating spatial patterns of cell function that follow from the process of founder cell specification during cleavage. Ultimately such diverse cell functions depend upon differential regulation of gene expression. Thus, a molecular interpretation of spatial diversification in the embryo must relate causally the process of founder cell specification, and the observed lineage-specific patterns of gene regulation.

\section{Materials and methods}

Sea urchin embryos were prepared for injection using a modification of the methods developed by McMahon and co-workers (1985). Briefly, Strongylocentrotus purpuratus gametes were obtained by injection of $0.5 \mathrm{M} \mathrm{KCl}$. Eggs are dejellied in $\mathrm{pH} 5.0$ seawater and semen was kept undiluted on ice. Eggs were bound by electrostatic charge to $60-\mathrm{mm}$ petri dish lids treated with $1 \%$ protamine sulfate. The eggs were fertilized with a freshly diluted sperm suspension and the embryos allowed to develop to the eight-cell stage.

Just before injection, the embryos were incubated in calciumand magnesium-free seawater for $5 \mathrm{~min}$ to soften the hyaline layer. Following the injection, calcium and magnesium ion concentrations were returned to those of artificial seawater, the dish was washed several times with Millipore-filtered seawater treated with penicillin (20 units/liter) and streptomycin (50 $\mu \mathrm{g} /$ liter), and the embryos were incubated in the dark for $72 \mathrm{hr}$.

Microinjection was accomplished using needles pulled on a Brown-Flaming horizontal needle puller (Sutter Instruments, San Rafael, California) from 1.0-mm o.d., omega-dot, borosilicate capillary tubing (Frederick Haer and Co., Brunswick, Maine). About $2 \mathrm{pl}$ of a solution containing dextran conjugated to fluorescein and lysine /Gimlich and Braun 1986; Molecular Probes, Inc., Eugene, Oregon) at a concentration of $40 \mathrm{mg} / \mathrm{ml}$ in $200 \mu \mathrm{M} \mathrm{KCl}$ and $10 \mathrm{~mm}$ phosphate buffer (pH 6.8) was injected into a single cell of each embryo.

At $72 \mathrm{hr}$ postfertilization, embryos were fixed by the addition of formaldehyde to a concentration of $4 \%$. Individuals positive for fluorescein staining were collected, photographed as whole mounts, and then embedded singly or in groups in agarose, followed by alcohol dehydration and paraffin embedding for light microscopy. Embryos were viewed on an Olympus BH2-RFL epifluorescence (excitation from the viewing side) photomicroscope and then photographed using Kodak VR400 Kodacolor film.

\section{Acknowledgments}

We thank Ronald Hill for technical assistance. Robert L. Gimlich kindly provided fluoresceinated dextran. This work was supported by National Science Foundation grant DCB 8544707 to R.A.C., by National Institutes of Health Grant HD-05753 to E.H.D., and by National Institutes of Health Biomedical Research Support grant RR07003. 


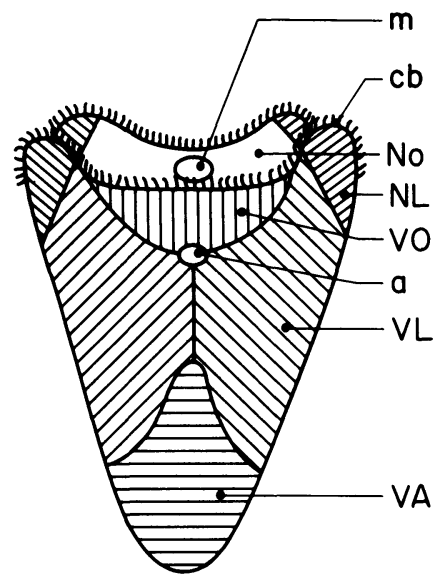

ANAL VIEW

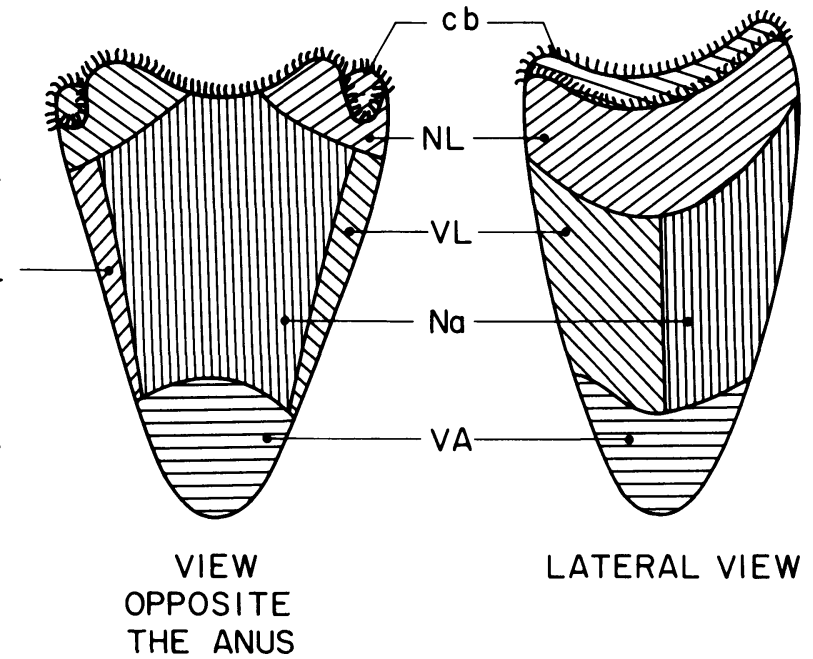

Figure 9. Regions of the larval ectoderm contributed by each of the blastomeres at the eight-cell stage. Symbols are as follows: (m) mouth; (cb) ciliated band; (a) anus.

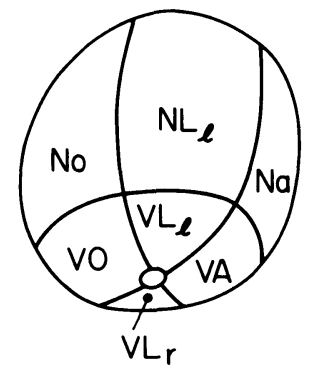

GASTRULA

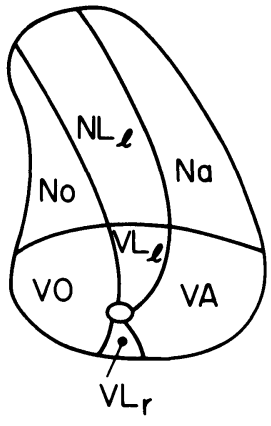

PRISM

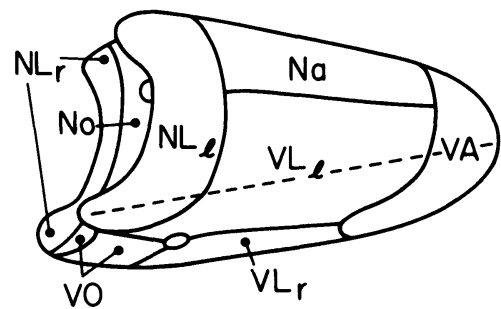

PLUTEUS

Figure 10. A diagram of the eight-cell blastomere fates mapped onto the gastrula, prism, and pluteus. In the prism, the elongating body rods transmit pressure to the region of the apical aboral ectoderm, carrying it to the vertex of the pluteus. The nearer body rod and anterolateral rod are represented by a dashed line in the pluteus.

\section{References}

Angerer, L.M., G. Kawczynski, D.G. Wilkinson, M. Nemer, and R.C. Angerer. 1986. Spatial patterns of metallothionein mRNA expression in the sea urchin embryo. Dev. Biol. 116: $543-547$.

Angerer, R.C. and E.H. Davidson. 1984. Molecular indices of cell lineage specification in sea urchin embryos. Science 226: $1153-1160$.

Benson, S., H.M. Sucov, L. Stephens, E.H. Davidson, and F. Wilt. 1987. A lineage specific gene encoding a major matrix protein of the sea urchin embryo spicule. Authentication of the cloned gene and developmental expression. Dev. Biol. (in press).

Boveri, T. 1901. Die Polarität von Oocyte, Ei und Larve des Strongylocentrotus lividis. Zool. Jahrb., Abt. Anat. Ontog. Tiere 14: 630-653.

Carson, D.D., M.C. Farach, D.S. Earles, G.L. Decker, and W.J. Lennarz. 1985. A monoclonal antibody inhibits calcium ac- cumulation and skeleton formation in cultured embryonic cells of the sea urchin. Cell 41: 639-648.

Cox, K.H., L.M. Angerer, J.J. Lee, E.H. Davidson, and R.C. Angerer. 1986. Cell lineage-specific programs of expression of multiple actin genes during sea urchin embryogenesis. $J$. Mol. Biol. 188: 159-172.

Czihak, G. 1963. Entwicklungsphysiologische Untersuchungen an Echiniden (Verteilung und Bedeutung der Cytochromoxydase). Wilhelm Roux's Archiv. Dev. Biol. Entwicklungsmech. Org. 154: 272-292.

Davidson, E.H. 1986. Gene activity in early development, 3rd ed. Academic Press, Orlando, Florida.

Dolecki, G.J., S. Wannakrairoj, R. Lum, G. Wang, H.D. Riley, R. Carlos, A. Wang, and T. Humphreys. 1986. Stage-specific expression of a homeobox containing gene in the nonsegmented sea urchin embryo. EMBO J. 5: 925-930.

Ettensohn, C.A. 1985. Gastrulation in the sea urchin embryo is accompanied by the rearrangement of invaginating epithelial cells. Dev. Biol. 112: 383-390. 
Floyd, E.E., Z. Gong, B.P. Brandhorst, and W.H. Klein. 1986. Calmodulin gene expression during sea urchin development: Persistence of a prevalent maternal protein. Dev. Biol. 113: 501-511.

Gibson, A.W. and R.D. Burke. 1985. The origin of the pigment cells in embryos of the sea urchin Strongylocentrotus purpuratus. Dev. Biol. 107: 414-419.

Gimlich, R.L. and J. Braun. 1986. Improved fluorescent compounds for tracing cell lineage. Dev. Biol. 109: 509-514.

Gustafson, T. and L. Wolpert. 1963. Studies on the cellular basis of morphogenesis in the sea urchin embryo. Exp. Cell Res. 29: 561-582.

Hardin, J.D. and L.Y. Chen. 1986. The mechanisms and mechanics of archenteron elongation during sea urchin gastrulation. Dev. Biol. 115: 490-501.

Harkey, M.A. and A.H. Whiteley. 1980. Isolation, culture and differentiation of echinoid primary mesenchyme cells. Wilhelm Roux's Arch. Dev. Biol. 189: 111-122.

Hörstadius, S. 1939. The mechanics of sea urchin development, studied by operative methods. Biol. Rev. Cambridge Philos. Soc. 14: 132-179.

-1973. Experimental embryology of echinoderms. Clarendon Press, Oxford.

Hörstadius, S. and A. Wolsky. 1936. Studien über die Determination der Bilateral-symmetrie des jungen Seeigel Keimes. Wilhelm Roux's Arch. Entwicklungsmech. Org. 135: 69113.

Lynn, D.A., L.M. Angerer, A.M. Bruskin, W.H. Klein, and R.C. Angerer. 1983. Localization of a family of mRNAs in a single cell type and its precursor in sea urchin embryos. Proc. Natl. Acad. Sci. 80: 2656-2660.

McClay, D.R., G.W. Cannon, G.M. Wessel, R.D. Fink, and R.B. Marchase. 1983. In Time, space, and pattern in embryonic development (ed. W.R. Jeffrey and R.A. Raff), pp. 157-169. Alan R. Liss, New York.

McMahon, A.P., C.N. Flytzanis, B.R. Hough-Evans, K.S. Katula, R.J. Britten, and E.H. Davidson. 1985. Introduction of cloned DNA into sea urchin cytoplasm: Replication and persistance during embryogenesis. Dev. Biol. 108: 420-430.

Okazaki, K. 1975. Normal development to metamorphosis. In The sea urchin embryo: Biochemistry and morphogenesis (ed. G. Czihak), pp. 177-232. Springer-Verlag, Berlin, New York.

Schroeder, T.E. 1980. Expression of the prefertilization polar axis in sea urchin eggs. Dev. Biol. 79: 428-443.

Snedecor, G.W. and W.G. Cochran. 1979. Statistical methods. Iowa State University Press, Ames, Iowa.

Sucov, H.M., S. Benson, J.J. Robinson, R.J. Britten, F. Wilt, and E.H. Davidson. 1987. A lineage specific gene encoding a major matrix protein of the sea urchin embryo spicule. Structure of the gene and derived sequence of the protein. Dev. Biol. (in press). 


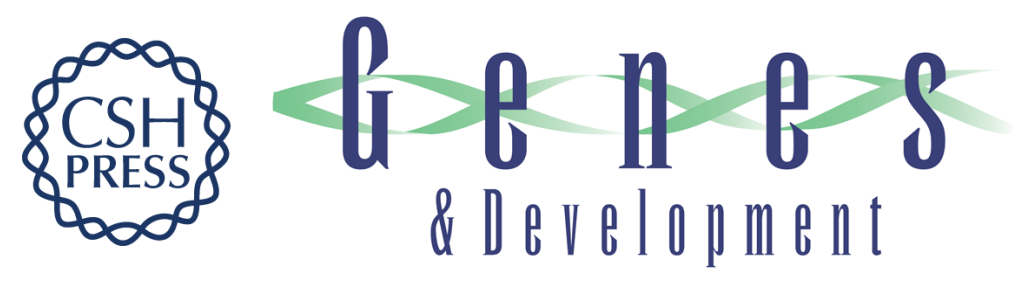

\section{Lineage and fate of each blastomere of the eight-cell sea urchin embryo.}

R A Cameron, B R Hough-Evans, R J Britten, et al.

Genes Dev. 1987, 1:

Access the most recent version at doi:10.1101/gad.1.1.75

References This article cites 19 articles, 2 of which can be accessed free at: http://genesdev.cshlp.org/content/1/1/75.full.html\#ref-list-1

\section{License}

Email Alerting

Receive free email alerts when new articles cite this article - sign up in the box at the top Service right corner of the article or click here.

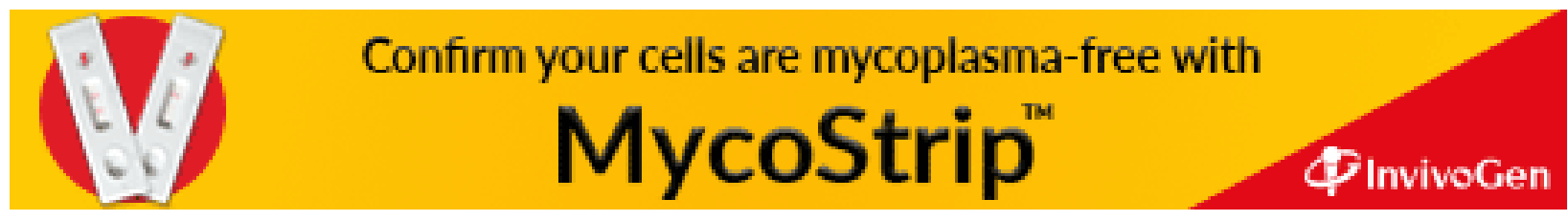

